\title{
A combination of mutations in the S1 part of the spike glycoprotein gene of coronavirus MHV-A59 abolishes demyelination
}

\author{
Li Fu, Donna M Gonzales, Jayasri Das Sarma, and Ehud Lavi \\ Division of Neuropathology, Department of Pathology and Laboratory Medicine, University of Pennsylvania, \\ School of Medicine, Philadelphia, Pennsylvania, USA
}

\begin{abstract}
The A59 strain of coronavirus, mouse hepatitis virus (MHV), produces acute hepatitis, meningoencephalitis, and chronic demyelination. The authors have previously shown that the spike (S) glycoprotein gene of MHV contains determinants of virulence, hepatitis, and demyelination. They then identified viruses containing mutations in the $\mathbf{S}$ gene that exhibit alterations in viral pathogenesis. In the present study, the authors produced new recombinant viruses with each one of these $S$ gene mutations by site-directed mutagenesis and targeted recombination and studied the effect of each individual mutation on the pathogenesis of the virus. They identified a combination of mutations in the S1 gene (I375M and L652I) that abolishes demyelination. Individual mutation and other combinations of mutations in the S gene only interfere with virulence and hepatitis and only reduce demyelination (I375M), but do not abolish demyelination completely. Thus, demyelination determinants exist within genomic regions on both sides of the hypervariable region, downstream from the receptor-binding domain in the S1 part of the MHV spike glycoprotein gene. The structure and precise function of these regions awaits further investigation. Journal of NeuroVirology (2004) 10, 41-51.
\end{abstract}

Keywords: coronaviruses; demyelination; mouse hepatitis virus; multiple sclerosis; nidoviruses

\section{Introduction}

Mouse hepatitis virus (MHV) is a member of the coronavirus genus, which belongs to the Coronaviridae family of the Nidovirales order (Cavanagh, 1997; Lai and Cavanagh, 1997). Nidoviruses pro-

Address correspondence to Dr. Ehud Lavi, University of Pennsylvania, School of Medicine, Division of Neuropathology, Department of Pathology and Laboratory Medicine, 613 StellarChance Laboratories, 422 Curie Boulevard, Philadelphia, PA 19104-6100, USA. E-mail: lavi@mail.med.upenn.edu

The present affiliation of Jayasri Das Sarma is Department of Physiology, University of Pennsylvania, School of Medicine, Philadelphia, Pennsylvania, USA.

This work has been presented in part at the annual meeting of the American Society for Virology in Madison, Wisconsin, July 2001. This work was supported in part by a National Multiple Sclerosis Society grant RG-2615 and a PHS grant NS30606. The authors thank Dr. Paul Masters for the use of viruses, plasmids, and cells; Dr. Thomas Gallagher for helpful discussions; and Elsa Aglow for excellent histologic expertise.

Received 21 April 2003; revised 3 September 2003; accepted 9 September 2003. duce a variety of hepatic, enteric, and neurologic diseases in animals, and upper respiratory and enteric diseases in humans (Lai and Cavanagh, 1997; Lavi et al, 1999; Lavi and Weiss, 1989). Infection of MHV-A59 in 4-week-old C57Bl/6 (B6) mice produces a biphasic disease with acute hepatitis and meningoencephalitis followed by chronic inflammatory demyelinating disease (Lavi et al, 1984, 1986). MHV infection in mice (with both A59 and JHM strains) serves as an excellent laboratory model for virus-induced demyelination, similar to multiple sclerosis (MS) in humans (Houtman and Fleming, 1996; Knobler et al, 1981; Lavi et al, 1984b; Perlman et al, 1990; Sorensen et al, 1984; Wege et al, 1982; Weiner, 1973). Because viral infection is considered as a potential trigger for demyelination in MS (Allen and Brankin, 1993), understanding the mechanism of MHV-induced demyelination and the upstream events that lead to the autoimmune phenomenon may provide important insight into the mechanism of interaction between viruses and the nervous system, especially with respect to 
the role of local and systemic immune system in viral neurotropism.

To understand the molecular basis of MHV neurotropism, we investigated a strain of MHV (MHV-2) that is closely related to MHV-A59 but is only weakly neurotropic (Hirano et al, 1974, 1981; Wege et al, 1981; Yamada et al, 1997; Yokomori et al, 1991). We sequenced the entire genome of MHV-2 and studied its detailed pathologic properties (Das Sarma et al, 2001b). We found that MHV-2 produces acute hepatitis and meningitis, without encephalitis, without demyelination, and without persistence of virus in the central nervous system (CNS) (Das Sarma et al, 2001b). We then replaced the $S$ gene of A59 with that of MHV-2 by targeted recombination and produced recombinant viruses that have a persistence-positive, demyelination-negative phenotype. We concluded that demyelination determinants, independent of viral persistence, map to the S gene of MHV (Das Sarma et al, 2000). Additional studies identified molecular determinants of pathogenesis in the S gene of MHV (Leparc-Goffart et al, 1998; Navas et al, 2001; Phillips et al, 1999, 2001).

To further explore which parts of the $\mathrm{S}$ gene are directly responsible for the demyelinating phenotype, we studied a set of recombinant viruses that were produced by infection with both MHV-2 and LA7, a temperature-sensitive (ts) mutant of MHV-A59 (Keck et al, 1988). These recombinant viruses were all found to be nondemyelinating. Sequencing the S genes of these recombinant viruses revealed that the recombinants' S gene was either derived from MHV2 or from MHV-A59. All the recombinant viruses with an S gene derived from MHV-A59 had three identical amino acid substitutions within the $S$ gene (Das Sarma et al, 2001a). Because these recombinant viruses were produced by dual infection of cultures with MHV-2 and LA-7, we could not rule out that they also contained other mutations in addition to the mutations in the S gene. We therefore used site-directed mutagenesis and targeted recombination to produce new recombinant viruses, with selective $S$ gene mutations that were associated with the demyelinationnegative phenotype.

\section{Results}

Generation of the $S$ gene recombinant viruses by targeted RNA recombination

We have previously identified three amino acid substitutions in the S gene (Figure 1) that are potentially associated with demyelination (I375M, L652I, and T1087N). By using the site-directed mutagenesis kit (see Materials and methods), we mutated three different sites individually or jointly. Targeted recombination was carried out between synthetic RNAs transcribed from recombinant vectors and recipient virus Alb4 (Penn99-1, 2) or fMHV (all other recombinants) according to previously described methods (Peng et al, 1995; Das Sarma et al, 2000). Alb4 is thermolabile and displays small plaque morphology at $39^{\circ} \mathrm{C}$; thus putative recombinants were selected as large plaque viruses after treatment for $24 \mathrm{~h}$ at $40^{\circ} \mathrm{C}$ and plating on L2 cell at $39^{\circ} \mathrm{C}$. The use of the virus with feline spike protein (fMHV) also provided a

\section{Structure of the MHV-A59 spike glycoprotein (S) gene}

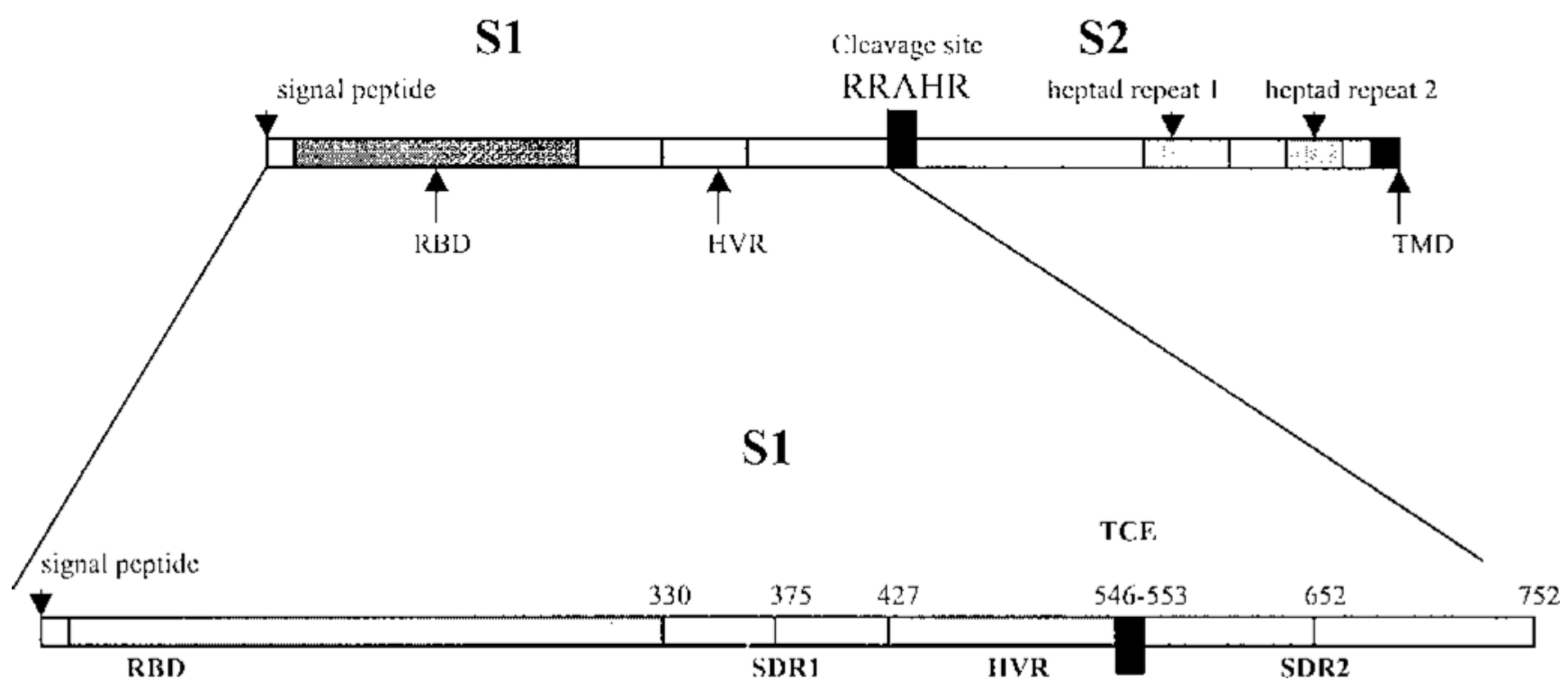

Figure 1 Schematic representation of the map of the spike glycoprotein gene with emphasis on the S1 portion of the gene. RBD, receptor binding domain; HVR, hypervariable region; TCE, CD8 ${ }^{+}$T-cell epitope H-2K $\mathrm{K}^{\mathrm{b}}$ : RCQIFANI; TMD, transmembrane domain; SDR1, $S$ demyelination region 1; SDR2, S demyelination region 2. 
useful method to select recombinant viruses against the background of parental viruses. Because fMHV does not grow in mouse cells, the recombination process with the transfected plasmid could be achieved in feline cells (FCWF). Recombinant viruses with S genes derived from mutated mouse $\mathrm{S}$ gene regained the ability to grow in mouse 17Cl-1 cells. By switching from feline cells to mouse cells that did not support the growth of parental $\mathrm{MMHV}$, we were able to select the recombinant viruses because only recombinant viruses with a mouse $S$ gene were present in these cultures. The potential recombinants were plaque purified twice and screened by using polymerase chain reaction (PCR) sequencing to detect the expected mutations made in the $\mathrm{S}$ gene. To verify that no secondary site mutations had been introduced into the $S$ gene, we sequenced the entire $S$ gene of the new recombinant viruses.

In vitro analysis of new recombinant viruses We analyzed the ability of the new recombinant viruses to grow in L2 cells by growth-curve kinetics analysis, and compared it with the MHV-A59 kinetics. We also performed growth analysis of the new viruses in different temperatures $\left(33^{\circ} \mathrm{C}, 37^{\circ} \mathrm{C}, 39^{\circ} \mathrm{C}\right)$ to rule out a ts phenotype. The growth kinetics of the viruses at $37^{\circ} \mathrm{C}$ is shown in Figure 2. It demonstrates that all viruses have similar growth properties. In addition, none of the viruses was ts.

In vivo analysis of new recombinant viruses

We analyzed the virulence and pathologic properties of the new recombinant viruses and compared it to that of wtMHV-A59 and the control recombinant virus wtR-A59. The virulence of the viruses is shown in Table 1. The T1087N mutation in S2 reduced viral virulence by fivefold. However, all other individual and combination of mutations in the $\mathrm{S} 1$ gene reduced

Viral Growth Curve in L2 Cells

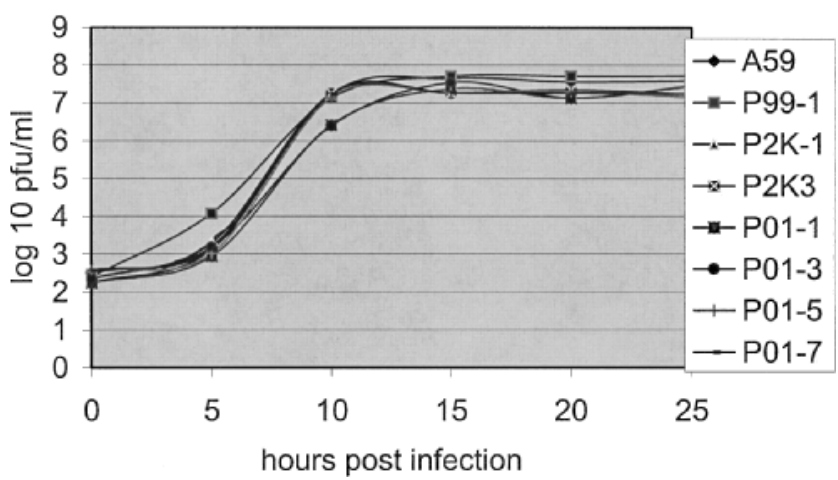

Figure 2 Viral titer kinetics of the newly constructed recombinant viruses in a 24-h analysis of samples from infected L2 cells, as evaluated by agarose-based plaque assays at $37^{\circ} \mathrm{C}$ on L2 cells following infection of $1 \mathrm{PFU}$ per cell $(\mathrm{m} . \mathrm{o.i}=1)$. Only one virus of each pair of identical recombinant viruses is shown. All viruses have growth patterns similar to MHV-A59.
Table 1 Virulence of recombinant viruses

\begin{tabular}{lr}
\hline Viruses & \multicolumn{1}{c}{$L D_{50}$} \\
\hline MHV-A59 & $4000 \mathrm{PFU}$ \\
WtR-A59 & $5000 \mathrm{PFU}$ \\
Penn2K-1, 2 (I375M, L652I, and T1087N) & $>50,000 \mathrm{PFU}$ \\
Penn2K-3, 4 (I375M and L652I) & $>50,000 \mathrm{PFU}$ \\
Penn01-1, 2 (I375M) & $>50,000 \mathrm{PFU}$ \\
Penn01-3, 4 (L652I) & $>50,000 \mathrm{PFU}$ \\
Penn99-1, 2 (T1087N) & $20,000 \mathrm{PFU}$ \\
Penn01-5, 6 (I375M and T1087N) & $>50,000 \mathrm{PFU}$ \\
Penn01-7, 8 (L652I and T1087N) & $>50,000 \mathrm{PFU}$ \\
\hline
\end{tabular}

virulence significantly (as assessed by $\mathrm{LD}_{50}$ ), and rendered the recombinant viruses nonlethal at the maximum titer of virus injected $(50,000$ plaque-forming units [PFU]). Reduced virulence correlated with reduction in the amount of hepatitis, mostly seen in the viruses that had mutations in the $\mathrm{S} 1$ gene.

The effect of the three mutations on the pathologic picture of encephalitis and hepatitis is shown in Figures 3 and 4 . Generally, none of the mutations or combination of mutations affected the extent of encephalitis. Hepatitis was abolished in all the viruses that contained the I375M mutation individually or with L652I (Penn01-1, 2; Penn2K-3, 4; and Penn2K-1, 2). Hepatitis was reduced in Penn01-3, 4 (L652I individual mutation), but interestingly, hepatitis was not significantly changed in combinations of the T1087N mutation with each one of the other S1 mutations (Penn01-5, 6 and Penn01-7, 8). The effect of the three mutations on viral replication in the infected organs (brain and liver) is shown in Figure 5. Viral titers in the brain and liver were consistent with the pathologic picture. Because viruses were injected into the brain, the brain titers on day 1 generally represent residual injected virus.

The effect of the three mutations on demyelination is shown in Table 2. Demyelination was not affected by the reduced virulence and most nonlethal viruses were still able to cause demyelination. Only viruses containing both I375M and L652I mutations in the S1 gene were nondemyelinating. Therefore, the two combinations that abolished demyelination were the one that included the I375M and L652I mutations (Penn2K-3 and Penn2K-4) and the one that included all three mutations I375M, L652I, and T1087N (Penn2K-1 and Penn2K-2). Neither one of these individual mutations was able to abolish demyelination completely. The I375M individual mutation and the combination of L652I and T1087N partially reduced demyelination, especially at the lower doses of viral injections. Interestingly, the combination of I375M and $\mathrm{T} 1087 \mathrm{~N}$ mutations did not reduce demyelination at the same level as the individual I375M mutation.

\section{Analysis of viral RNA persistence}

Viral RNA persistence of all recombinant and parental viruses in mouse organs (brain, spinal cord and liver) was analyzed by reverse transcriptase 
MHV-A59

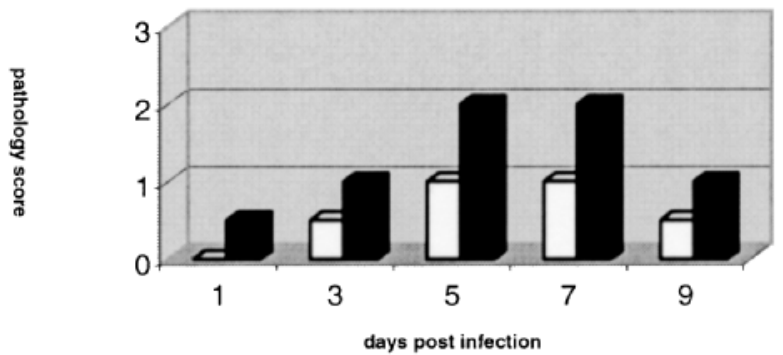

Penn99-1

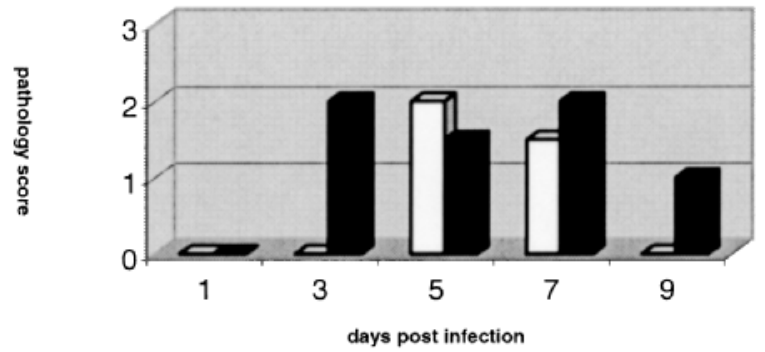

Penn01-1

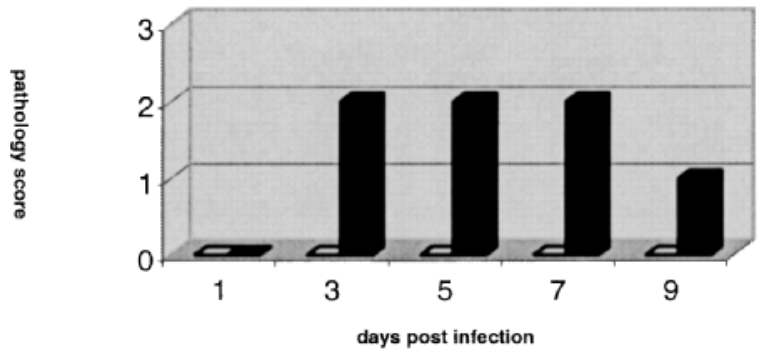

Penn01-3

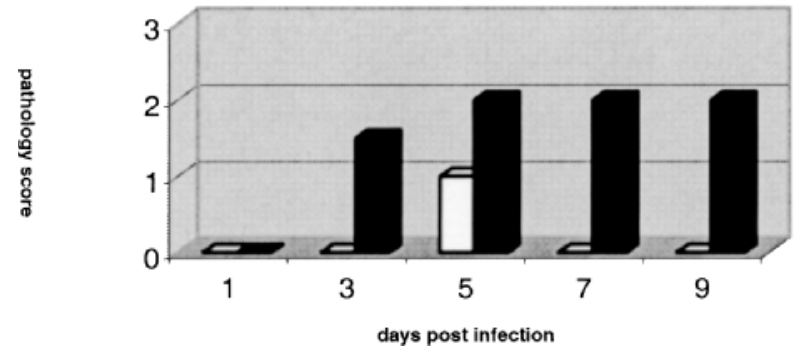

Penn2K-3

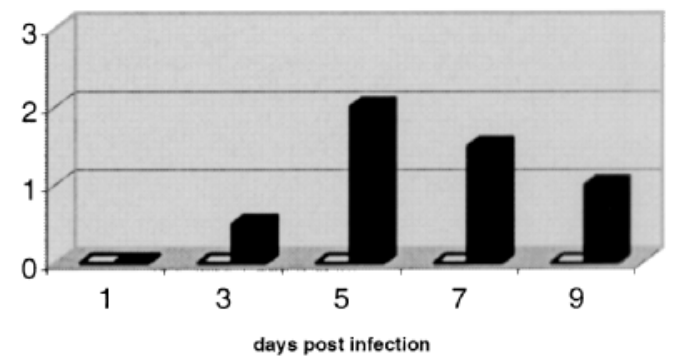

Penn01-5

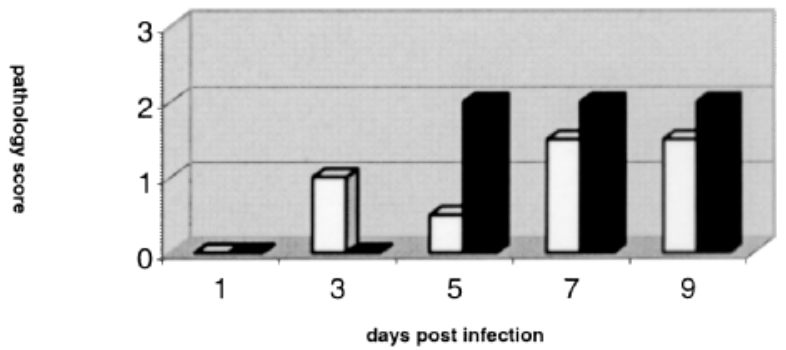

Penn01-7

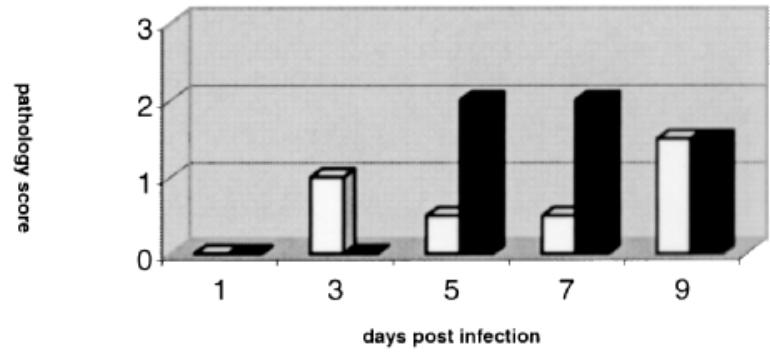

Penn2K-1

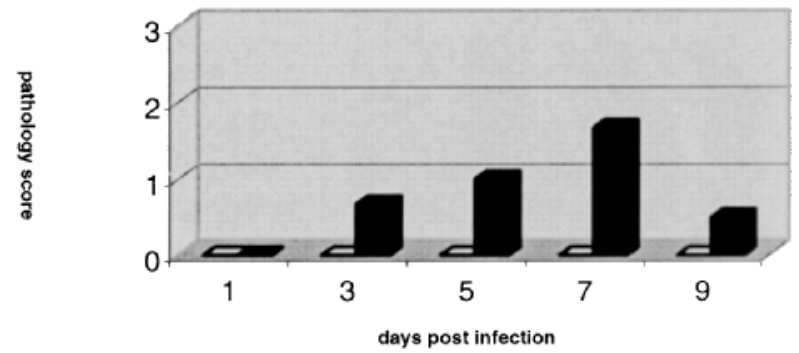

Figure 3 Pathologic analysis of recombinant viruses during acute infection. Mice were injected IC with 1000 PFU per mouse with each virus. Only one recombinant virus from each set of duplicate viruses is shown; however, similar findings were found for both viruses. Each time point represents the pathologic analysis of two to three mice. Dark bars represent encephalitis whereas white bars represent hepatitis. The intensity of the pathologic damage of encephalitis was based on a 3-level scale: $0=$ no inflammatory infiltrates in the brain; 1 = focal inflammatory infiltrate; 2 = multifocal inflammatory infiltrates; 3 = diffuse inflammatory infiltrates in the entire brain (panencephalitis). The intensity of the hepatitis was evaluated based on a 4-level scale: $0=$ no hepatitis; $1=$ one to two foci of necrosis in each section of liver; $2=$ three to six necrotic foci in each liver section; $3=$ more than six foci of nonbridging necrosis in each liver section; $4=$ diffuse bridging necrosis of the liver with islands of preserved parenchyma. 

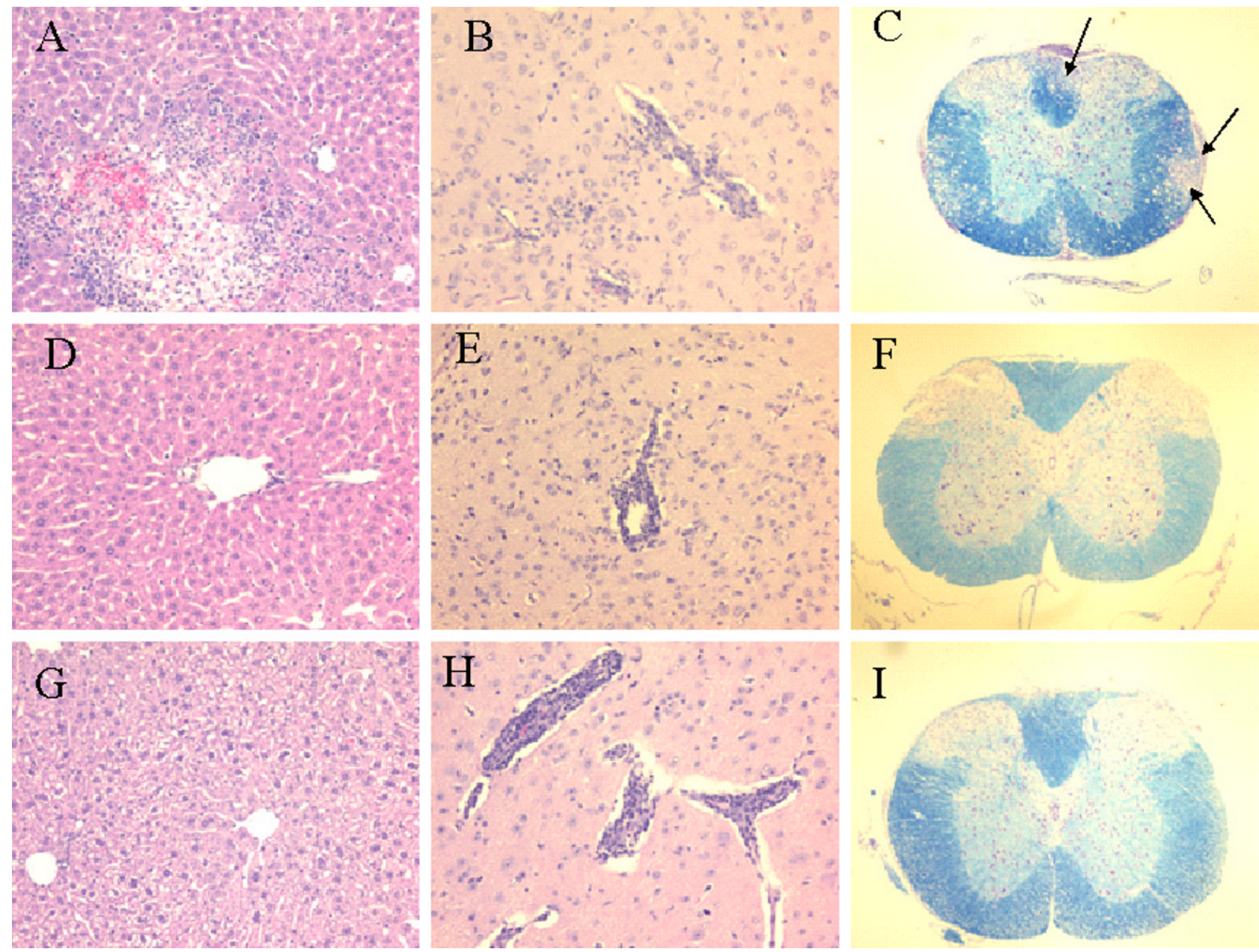

Figure 4 Histopathology of mouse organs following infection with MHV-A59 and demyelination-negative recombinant viruses. (A) Fourweek-old C57Bl/6 mice were injected IC with 1000 PFU of MHV-A59 and sacrificed 5 days post infection. Liver section shows necrotic lesions with cellular debris, inflammatory cells (polymorphonuclear leukocytes, lymphocytes and macrophages), and focal hemorrhage, representing acute hepatitis (H\&E × 200). (B) Four-week-old C57Bl/6 mice were injected IC with 1000 PFU of MHV-A59 and sacrificed 5 days post infection. Brain section shows perivascular lymphocytic infiltration microglial proliferation and pyknotic neurons representing acute encephalitis (H\&E × 200). (C) Four-week-old C57Bl/6 mice were injected IC with 5000 PFU of the MHV-A59 and sacrificed 30 days post infection. Spinal cord section shows multiple inflammatory demyelinating lesions in the white matter of the posterior and lateral columns (Luxol-Fast-Blue and cresyl violet $\times 50$ ). (D) Four-week-old C57Bl/6 mice were injected IC with 1000 PFU of Penn2K-1 and sacrificed 5 days post infection. Liver section shows normal histology (H\&E $\times 200)$. (E) Four-week-old C57Bl/6 mice were injected IC with 1000 PFU of Penn2K-1 and sacrificed 5 days post infection. Brain section shows perivascular lymphocytic infiltration microglial proliferation and pyknotic neurons representing acute encephalitis $(\mathrm{H} \& \mathrm{E} \times 200)$. (F) Four-week-old C57Bl/6 mice were injected IC with 5000 PFU of the Penn2K-1 and sacrificed 30 days post infection. Spinal cord section shows normal histology (Luxol-Fast-Blue and cresyl violet $\times 40$ ). (G) Four-week-old C57Bl/6 mice were injected IC with 1000 PFU of Penn2K-3 and sacrificed 5 days post infection. Liver section shows normal histology (H\&E $\times 200)$. (H) Four-week-old C57Bl/6 mice were injected IC with 1000 PFU of Penn2K-3 and sacrificed 5 days post infection. Brain section shows perivascular lymphocytic infiltration microglial proliferation and pyknotic neurons representing acute encephalitis $(\mathrm{H} \& \mathrm{E} \times 200)$. (I) Four-week-old C57Bl/6 mice were injected IC with 5000 PFU of the Penn2K-3 and sacrificed 30 days post infection. Spinal cord section shows normal histology (Luxol-Fast-Blue and Cresyl Violet $\times 40$ ).

(RT)-PCR. The results of RNA persistence in the spinal cord are shown in Figure 6. Both MHVA59 and recombinant virus wtR-A59 showed a prominent band consistent with MHV RNA. Similar bands were detected following infections with Penn99-1, Penn01-1, Penn01-3, and Penn01-5, all of which are demyelinating viruses. The demyelinatingnegative virus Penn2K-1 showed no RNA persistence in the cord; however, the other demyelinatingnegative virus Penn2K-3 showed a band similar to demyelinating-positive viruses. The demyelinationpositive virus Penn01-7 showed a weaker positive band. Thus, although some degree of RNA persistence may be required for demyelination, the presence of persistence is insufficient for the development of demyelination. RNA persistence in the brain was similar to the spinal cords but with a slightly weaker signal. RNA persistence in the liver was minimal and was only detected in MHV-A59 and wtR-A59 infections (data not shown). 

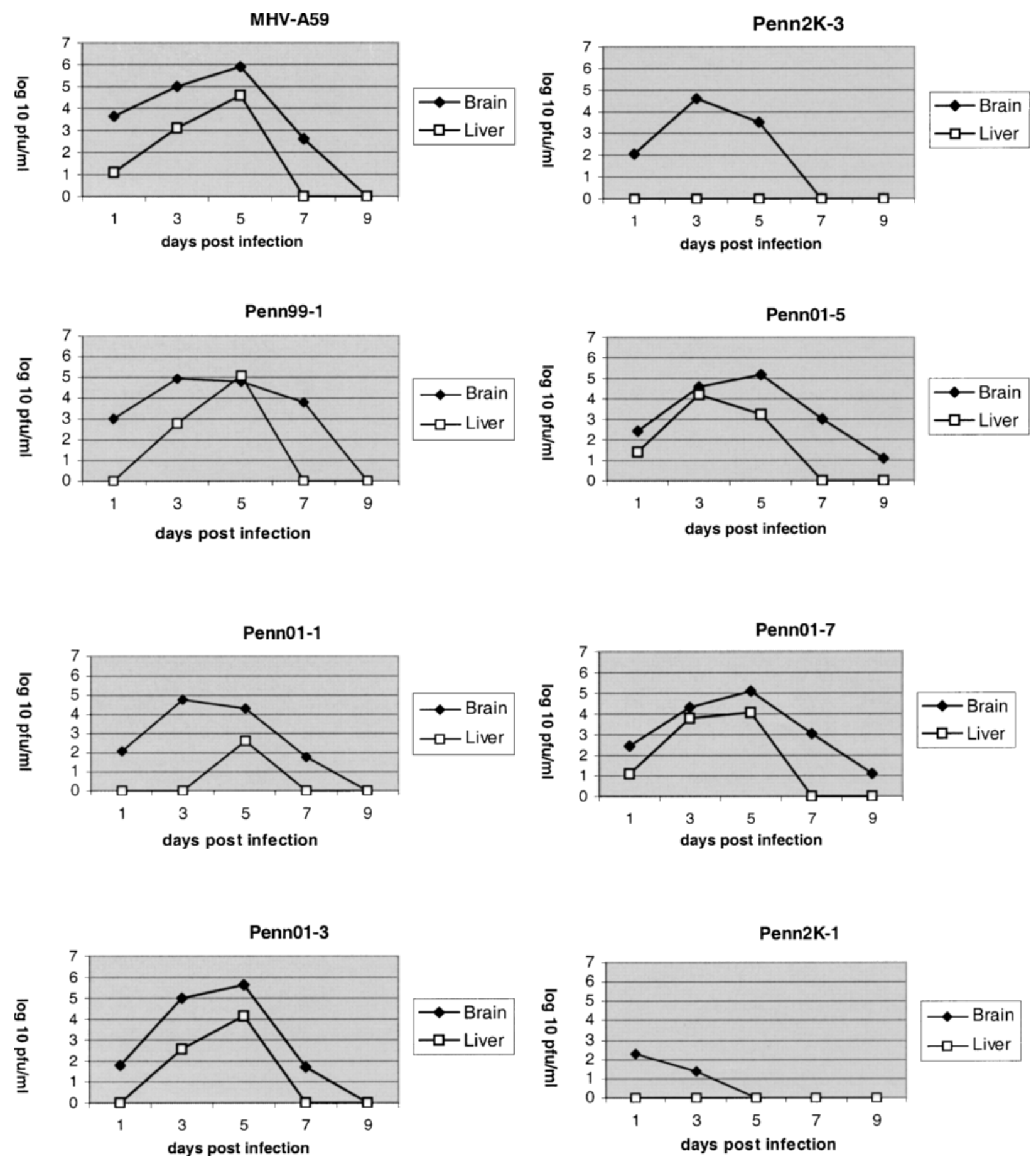

Figure 5 Viral growth kinetics or new recombinant viruses in liver and brains of infected mice. Mice were injected IC with $1000 \mathrm{PFU}$ per mouse with each virus. Each time point represents the mean titer analysis of two to three mice. Data present log 10 PFU $/ \mathrm{ml}$ of $w / v$ organ homogenates. Only one recombinant virus from each set of duplicate viruses is shown; however, similar findings were found for both viruses. Samples of liver and brain from infected mice at different time points post infection were tested by plaque assay on L2 cells.

\section{Discussion}

The MHV genome is the largest among RNA viruses. Although the generation of an infectious MHV clone has been recently reported (Yount et al, 2002), the new construct appears to be attenuated in vivo. Thus, molecular pathogenesis studies continue to require a complex manipulation of the MHV genome in order to introduce targeted changes into specific locations. To date, the best technique available for this purpose is targeted $3^{\prime}$ end heterologous recombination, originally introduced by Paul Masters (Peng et al, 1995). 
Table 2 Demyelinating properties of recombinant viruses

\begin{tabular}{|c|c|c|c|c|c|c|}
\hline \multirow[b]{2}{*}{ Viruses } & \multicolumn{3}{|c|}{ No. of mice with demyelination/total no. of mice (\%) } & \multicolumn{3}{|c|}{ Demyelinated quadrants/total number of quadrants (\%) } \\
\hline & $500 \mathrm{PFU}$ & 4000-5000 PFU & $50,000 \mathrm{PFU}$ & $500 \mathrm{PFU}$ & 4000-5000 PFU & 50,000 PFU \\
\hline MHV-A59 & $5 / 5(100 \%)$ & $9 / 9(100 \%)$ & N/A & $33 / 84(39 \%)$ & $98 / 128(77 \%)$ & N/A \\
\hline Penn99-2 & $8 / 8(100 \%)$ & $6 / 6(100 \%)$ & $2 / 2(100 \%)$ & $27 / 44(61 \%)$ & $32 / 52(62 \%)$ & 8/12 (67\%) \\
\hline Penn01-1 & $3 / 9(33 \%)$ & $2 / 10(20 \%)$ & $9 / 10(90 \%)$ & $7 / 160(4 \%)$ & $2 / 184(1 \%)$ & $24 / 188(13 \%)$ \\
\hline Penn01-3 & $10 / 10(100 \%)$ & $10 / 10(100 \%)$ & $10 / 10(100 \%)$ & $50 / 168(30 \%)$ & $54 / 184(30 \%)$ & $64 / 200(32 \%)$ \\
\hline Penn2K-3 & $0 / 10(0 \%)$ & $0 / 10(0 \%)$ & $1 / 10(10 \%)$ & 0/116 (0\%) & $0 / 120(0 \%)$ & $1 / 120(0.8 \%)$ \\
\hline Penn01-5 & $10 / 10(100 \%)$ & $9 / 10(90 \%)$ & $10 / 10(100 \%)$ & $83 / 200(42 \%)$ & $33 / 111(30 \%)$ & $97 / 148(66 \%)$ \\
\hline Penn01-7 & $3 / 5(60 \%)$ & $1 / 5(20 \%)$ & $10 / 10(100 \%)$ & $12 / 100(12 \%)$ & $21 / 96(22 \%)$ & $36 / 200(18 \%)$ \\
\hline Penn2K-1 & $0 / 10(0 \%)$ & $0 / 10(0 \%)$ & $0 / 10(0 \%)$ & $0 / 120 \quad(0 \%)$ & $0 / 124(0 \%)$ & $0 / 100(0 \%)$ \\
\hline
\end{tabular}

Note. MHV-A59 and representative recombinant viruses of each pair were analyzed for their ability to cause spinal cord demyelination following IC injections with three different doses of the virus. The other virus of each pair presented a similar phenotype. MHV-A59 was not evaluated at the highest dose because there are no surviving mice at this dose on day 30 post infection.

We used this technique in our previous studies, in which we identified the demyelination determinants in the $\mathrm{S}$ gene (Das Sarma et al, 2000). We continued our investigation in the present study to better define the regions of genomic control of demyelination within the S gene.

Although incidental mutations during coronavirus replication are not uncommon, we used strict precautions to reduce the chance that incidental mutations might interfere with molecular pathogenesis studies. We sequenced the entire $S$ gene of each new recombinant virus immediately before it was used in pathogenesis studies to confirm the presence of the desired mutations and to exclude the possibility of incidental undesired mutations. The pathogenesis of new recombinant viruses was tested immediately after the generation of the viruses to avoid unnecessary passages of the viruses in culture, which tend to increase the chance for mutations. We generated two identical recombinant viruses for each mutation in two independent recombination procedures. Both viruses were tested in mice to ensure an identical in vivo phenotype. These precautions reduced the chance for incidental mutations to a negligible level.

Using these principles, we found that a combination of mutations in the S1 gene abolishes demyelination only when introduced simultaneously. The I375M mutation, which appears to be the dominant and the more important one, reduces the amount of demyelination but does not abolish it completely. However, I375M in combination with L652I completely abolishes demyelination. Thus, although this may not be the only genomic site that controls demyelination, the region in the S1 gene downstream from the receptor-binding domain contains important determinants of demyelination (Figure 1).
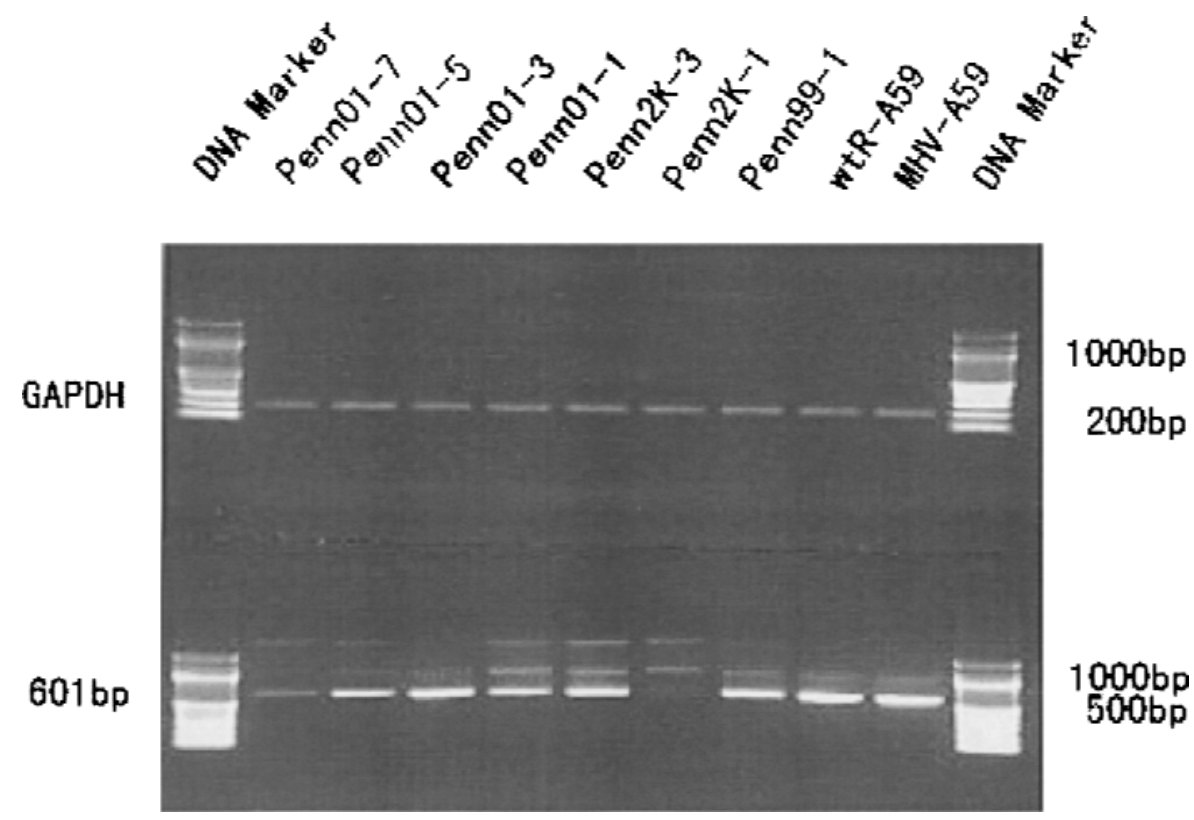

Figure 6 RT-PCR analysis of viral RNA persistence in the spinal cord of mice 30 days post infection with recombinant and parental viruses. 
The coronavirus spike protein is a type I membrane protein with approximately a 1300-residue ectodomain, an 18-residue transmembrane portion, and a 38-residue cytoplasmic tail (Schmidt et al, 1987). The full-length spike glycoprotein oligomerizes rapidly after synthesis and then cleaves by a furin-like protease into two similar-sized fragments: a peripheral S1 and a membrane-anchored S2 (Sturman et al, 1985). S1 and S2 are associated by noncovalent interactions. S1 is responsible for binding to cellular receptors whereas S2 has the primary responsibility for membrane fusion. The receptors for MHV are members of the family of carcinoembryonic antigen cell adhesion molecules (CEACAM), of the immunoglobulin superfamily (Dveksler et al, 1991, 1993a, 1993b). Dimers of S1 bind to a single molecule of CEACAM. The binding sites on S1 are presumably juxtaposed, causing a steric hindrance or global conformational changes that preclude additional interactions (Lewicki and Gallagher, 2002). After binding to receptor, the spike protein undergoes conformational changes, including alternative disulfide linkages and separation of S1 from S2, which may increase the propensity of $S$ to fuse membranes (Taguchi and Matsuyama, 2002).

We hypothesize that the I375M and L652I mutations mark potential S1 genomic sites that control demyelination. These mutations are in the middle of two regions of yet undesignated name or function on either side of the hypervariable region of S1. As shown in Figure 1, we provisionally labeled these regions as $\mathrm{S}$ demyelination region 1 (SDR1) and $\mathrm{S}$ demyelination region 2 (SDR2). There are no known T- or B-cell epitopes in these regions; however, these $S 1$ regions may influence spike protein functions in several ways. Binding of S1 to receptor is unlikely to be significantly affected by sites outside the first 330 amino acids because the CEACAM binding site can exist wholly in the amino terminal 330 residues of S1. However, SDR1 and SDR2 may still have influence on S1-CEACAM binding kinetics, which may affect pathogenesis. Similarly, the SDR1 and SDR2 regions may alter the kinetics and/or extent of the fusion activity of the spike protein, or the kinetics and/or extent of separation of S1 from S2. Either one of these changes may have an effect on the pathogenesis of the virus. The fact that two mutations are necessary for the abolishment of demyelination may rely on the three-dimensional spatial structure and folding of the S1 part of the molecule and on potential chemical interactions between the two sites.

Both I375M and L652I mutations also reduce hepatitis and therefore reduce the overall virulence of the viruses resulting in a nonlethal phenotype. However, the reduced virulence per se is not enough to abolish demyelination. We have shown here and in a previous publications (Das Sarma et al, 2000, 2001a, 2001b) that viruses that lack the ability to produce demyelination can be either less virulent (Penn2K-1, and Penn2K-2) or significantly more vir- ulent (Penn98-1 and Penn98-2, and MHV-2) than MHV-A59. Moreover, the individual L652I mutation has a nonlethal phenotype but no effect on demyelination. In addition, ability of the virus to produce encephalitis may be a prerequisite, but is insufficient for the development of subsequent demyelination. For example, Penn2K-3 and penn2K-4 produce encephalitis similar to demyelinating viruses but do not produce demyelination. This study, therefore, provides further evidence that the ability of a virus to produce chronic demyelination is not in direct correlation with the virulence of the virus. Similarly, viral persistence may be a prerequisite but is insufficient for the development of demyelination. The property of demyelination is therefore a unique additional neurotropic property, which appears to be at least in part encoded by the S1 gene of the MHV genome.

\section{Materials and methods}

\section{Viruses and cells}

The following plaque-purified viruses were used in this study: MHV-A59 (Budzilowicz et al, 1985; Lavi et al, 1984a, 1984b), Alb4 (obtained from Paul Masters, Albany, NY), and fMHV (Kuo et al, 2000) (obtained from Paul Masters, Albany, NY). Stock viruses had titers of $10^{7}$ to $10^{8} \mathrm{PFU} / \mathrm{ml}$. Viruses were propagated and titered on murine L2 cells, 17Cl-1 cells, and feline FCWF cells in Dulbecco's minimum essential medium (DMEM) with 10\% fetal bovine serum (FBS).

\section{Plasmids and PCR mutagenesis}

The pMH54 plasmid (obtained from Paul Masters) contains the 1.1-kb, $3^{\prime}$ end of MHV-A59 genome from the HE gene downstream (Das Sarma et al, 2000; Kuo et al, 2000). The sequence of the $S$ gene in pMH54 is identical to that of MHV-A59 except for the introduction of AvrII and sbfI restriction sites, which allowed the insertion of different $S$ genes into the background of pMH54, and enabled the identification of recombinant viruses containing full length recombinant S gene. Site-directed mutagenesis experiments were performed using the Stratagene QuickChange kit (Cedar Creek, TX).

\section{Targeted RNA recombination and selection of recombinants}

Targeted RNA recombinations were carried out between Alb4 (for Penn99-1, 2), fMHV (for all other recombinant viruses), and synthetic RNAs. Alb4 is thermolabile and displays small plaque morphology at $39^{\circ} \mathrm{C}$. fMHV contains an $S$ gene from FIPV on an MHV-A59 background (Kuo et al, 2000) and can only grow in feline cells FCWF. The synthetic RNAs were supplied by the pMH54 plasmid containing the entire $3^{\prime}$ end of the MHV genome from gene 2 (HE) to 7 (N). For recombination, L2 cells spinner culture (for Penn99-1, 2 only) or FCWF (all other recombinants) 
was harvested and infected at multiplicity of infection (m.o.i) $=1$ of Alb4 (at $33^{\circ} \mathrm{C}$ ), or fMHV (at $37^{\circ} \mathrm{C}$ ), respectively. Cells were then transfected by electroporation with the synthetic RNA by using two consecutive pulses from Bio-Rad Gene Pulser apparatus set at $0.3 \mathrm{kV}$ and $960 \mu \mathrm{F}$. Infected and transfected cells were plated onto a monolayer of $17 \mathrm{Cl}-1$ cells, and the released viruses were harvested at approximately 24 to $48 \mathrm{~h}$ post infection. For selection of the recombinant viruses, a two-step plaque purification was performed and PCR sequencing of the $S$ gene was used to confirm the presence of the desired mutations. For each mutation, we produced two identical viruses in two independent recombination events. This strategy was designed to reduce the possibility that random unrecognized mutations would affect the phenotype. The new recombinant viruses were designated as the following (also in Table 1): (1) Penn2K1 and Penn2K2 contain an MHV-A59 S gene with the I375M, L652I, and T1087N 3-point mutations; (2) Penn2K3 and Penn2K4 contain an MHV-A59 S gene with the I375M and L652I 2-point mutations; (3) Penn99-1 and Penn99-2 contain an MHV-A59 S gene with the T1087N single-point mutation (Das Sarma et al, 2001c); (4) Penn01-1 and Penn01-2 contain an MHV-A59 S gene with the I375M single-point mutation; (5) Penn01-3 and Penn01-4 contain an MHV-A59 S gene with the L652I single-point mutation; (6) Penn01-5 and Penn016 contain an MHV-A59 S gene with the I375M and T1087N two-point mutations; (7) Penn01-7 and Penn01-8 contain an MHV-A59 S gene with the L652I and T1087N 2-point mutations. Finally, a control virus, wtR-A59, was generated by targeted recombination in which the S gene of MHV-A59 was recombined back into the MHV-A59 background, to eliminate the possibility that any changes in pathogenesis might derive from the recombination process itself.

\section{$S$ gene sequencing}

For sequencing of the S gene, RT-PCR was carried out on templates of cytoplasmic RNA extracted from virus-infected L2 cells. Double-stranded PCR products were analyzed by automated sequencing using the Taq dye terminator procedure, with previously described primers, according to the manufacturer's protocol (Das Sarma et al, 2000, 2001a, 2001b).

\section{Viral RNA persistence assay}

Mice (C57BL/6) were infected intracerebrally with $1000 \mathrm{PFU}$ of each of the recombinant viruses along with same dose MHV-A59 and wtR-A59. Two mice per virus were analyzed independently 30 days post infection. Briefly, mice were sacrificed and perfused intracardially with $10 \mathrm{ml}$ phosphate-buffered saline (PBS). Liver, brain and spinal cord were removed, cleaned with PBS, then snap-frozen with liquid nitrogen. Tissue RNA was isolated with RNeasy Mini kit (QIAGEN). After quantification, $1 \mu \mathrm{g}$ of each RNA sample was re- verse transcribed with SuperScript RT-PCR system (Invitrogen) to synthesis first strand cDNA. PCR was performed with the oligonucleotide primers IZJ5 (5'-GCTCCAACAGTTGGTGCC-3') and IZJ6 (5'-ACGTAGGACCTTGCTAACTTC- $3^{\prime}$ ). The primers IZJ5 and IZJ6 were designed to detect the most conserved region of MHV-A59 N gene and the $3^{\prime}$ untranslated region. A 601-bp PCR product was analyzed by agarose gel electrophoresis.

\section{Viral growth curves}

L2 cell cultures were prepared in 12-well plates in DMEM with $10 \%$ FBS. Confluent monolayers were infected with each virus (m.o.i. = 1) in duplicate wells and incubated for $1 \mathrm{~h}$ at $37^{\circ} \mathrm{C}$. After adsorption, the cells were washed with PBS three times and then fed with $1.5 \mathrm{ml}$ medium. At the indicated times, the cells were lysed by three cycles of freeze-thawing, and the supernatants were removed and titered by plaque assay on L2 cells as previously described (Lavi et al, 1984b).

\section{Mice and virulence assays}

All animal experiments used 4-week-old virus-free C57BL/6 mice (NCI; Bethesda, MD). Mice were anesthetized with methofane. The amount of virus inoculated by in each experiment was diluted in PBS containing $0.75 \%$ bovine serum albumin (BSA) and a total volume of $25 \mu \mathrm{l}$ was injected into the left cerebral hemisphere (IC). Virulence assays (LD50 dose) were performed as previously described by IC inoculation of 10 mice per dilution with 10 -fold serial dilutions of wild-type or recombinant viruses (Lavi et al, 1984b). Mice were examined for signs of disease or death on a daily basis for up to 30 days post infection.

\section{Histologic analysis}

For organ analysis, mice were sacrificed at various time points post infection. After perfusion with PBS, brains and livers were removed. Half of the brain and a portion of liver were fixed in $10 \%$ buffered formalin, paraffin-embedded, and stained with hematoxylin and eosin (H\&E) for a blinded histologic analysis. Samples of brain and liver were frozen in $-80^{\circ} \mathrm{C}$, to be used later for assessment of viral titers by plaque assays. For assessment of demyelination, mice were sacrificed at 30 days post infection. Mice were perfused with PBS followed by $10 \%$ buffered formalin. Spinal cords were removed, formalin-fixed, and paraffin embedded. Four to six transverse sections of cervical, thoracic, and lumbar levels of the cord were stained with Luxol fast blue (LFB) and the number of quadrants of cord containing demyelination were counted as a fraction of the total number of cord quadrants evaluated in each individual mouse (Lavi et al, 1984b, 1986, 1988, 1990). 


\section{References}

Allen I, Brankin B (1993). Pathogenesis of multiple sclerosis-the immune diathesis and the role of viruses. J Neuropathol Exp Neurol 52: 95-105.

Budzilowicz CJ, Wilczynski SP, Weiss SR (1985). Three intergenic regions of mouse hepatitis virus strain A59 genome RNA contain a common nucleotide sequence that is homologous to the $3^{\prime}$ end of the viral mRNA leader sequence. J Virol 53: 834-840.

Cavanagh D (1997). Nidovirales: a new order comprising Coronaviridae and Arteriviridae. Arch Virol 142: 629633.

Das Sarma J, Fu L, Hingley ST, Lai MM, Lavi E (2001a). Sequence analysis of the $S$ gene of recombinant $\mathrm{MHV}$ 2/A59 coronaviruses reveals three candidate mutations associated with demyelination and hepatitis. J NeuroVirol 7: 432-436.

Das Sarma J, Fu L, Hingley ST, Lavi E (2001b). Mouse hepatitis virus type-2 infection in mice: an experimental model system of acute meningitis and hepatitis. Exp Mol Pathol 71: 1-12.

Das Sarma J, Fu L, Lavi E (2001c). The effect of the T1087N $S$ gene mutation on MHV-A59 pathogenesis. Adv Exp Med Biol 494: 155-157.

Das Sarma J, Fu L, Tsai JC, Weiss SR, Lavi E (2000). Demyelination determinants map to the spike glycoprotein gene of coronavirus mouse hepatitis virus. J Virol 74: 92069213.

Dveksler GS, Dieffenbach CW, Cardellichio CB, McCuaig K, Pensiero MN, Jiang GS, Beauchemin N, Holmes KV (1993a). Several members of the mouse carcinoembryonic antigen-related glycoprotein family are functional receptors for the coronavirus mouse hepatitis virus-A59. J Virol 67: 1-8.

Dveksler GS, Pensiero MN, Cardellichio CB, Williams RK, Jiang G-S, Holmes KV, Dieffenbach CW (1991). Cloning of mouse hepatitis virus (MHV) receptor: expression in human and hamster cell lines confers susceptibility to MHV. J Virol 65: 6881-6891.

Dveksler GS, Pensiero MN, Dieffenbach CW, Cardellichio CB, Basile AA, Elia PE, Holmes KV (1993b). Mouse hepatitis virus strain A59 and blocking antireceptor monoclonal antibody bind to the N-terminal domain of cellular receptor. Proc Natl Acad Sci U S A 90: 1716-1720.

Hirano N, Fujiwara K, Hino S, Matumoto M (1974). Replication and plaque formation of mouse hepatitis virus (MHV-2) in mouse cell line DBT culture. Archiv Ges Virusfors 44: 298-302.

Hirano N, Murakami T, Taguchi F, Fujiwara K, Matumoto $M$ (1981). Comparison of mouse hepatitis virus strains for pathogenicity in weanling mice infected by various routes. Arch Virol 70: 69-73.

Houtman JJ, Fleming JO (1996). Pathogenesis of mouse hepatitis virus-induced demyelination. J NeuroVirol 2: 361376.

Keck JG, Soe LH, Makino S, Stohlman SA, Lai MMC (1988). RNA recombination of murine coronavirus: recombination between fusion-positive mouse hepatitis virus A59 and fusion-negative mouse hepatitis virus 2. J Virol 62: 1989-1998.

Knobler RL, Haspel MV, Oldstone MBA (1981). Mouse hepatitis virus type 4 (JHM strain)-induced fatal central nervous system disease, part 1 (genetic control and the murine neuron as the susceptible site for disease). J Exp Med 153: 832-843.
Kuo L, Godeke GJ, Raamsman MJ, Masters PS, Rottier PJ (2000). Retargeting of coronavirus by substitution of the spike glycoprotein ectodomain: crossing the host cell species barrier. J Virol 74: 1393-1406.

Lai MMC, Cavanagh D (1997). The molecular biology of coronaviruses. Adv Virus Res 48: 1-100.

Lavi E, Fishman SP, Highkin MK, Weiss SR (1988). Limbic encephalitis following inhalation of murine coronavirus MHV-A59. Lab Invest 58: 31-36.

Lavi E, Gilden DH, Highkin MK, Weiss SR (1984a). Persistence of MHV-A59 RNA in a slow virus demyelinating infection in mice as detected by in situ hybridization. J Virol 51: 563-566.

Lavi E, Gilden DH, Highkin MK, Weiss SR (1986). The organ tropism of mouse hepatitis virus A59 is dependent on dose and route of inoculation. Lab Anim Sci 36: 130135.

Lavi E, Gilden DH, Wroblewska Z, Rorke LB, Weiss SR (1984b). Experimental demyelination produced by the A59 strain of mouse hepatitis virus. Neurology 34: 597603.

Lavi E, Murray EM, Makino S, Stohlman SA, Lai MM, Weiss SR (1990). Determinants of coronavirus MHV pathogenesis are localized to $3^{\prime}$ portions of the genome as determined by ribonucleic acid-ribonucleic acid recombination. Lab Invest 62: 570-578.

Lavi E, Schwartz T, Jin YP, Fu L (1999). Nidovirus infections: experimental model systems of human neurologic diseases. J Neuropathol Exp Neurol 58: 1197-1206.

Lavi E, Weiss SR (1989). Coronaviruses. In: Clinical and molecular aspects of neurotropic viral infections. Gilden DH, Lipton HL (eds). Boston, MA: Kluwer, Academic Publishers, pp 101-139.

Leparc-Goffart I, Hingley ST, Chua MM, Phillips J, Lavi E, Weiss SR (1998). Targeted recombination within the spike gene of murine coronavirus mouse hepatitis virusA59: Q159 is a determinant of hepatotropism. J Virol 72: 9628-9636.

Lewicki DN, Gallagher TM (2002). Quaternary structure of coronavirus spikes in complexwith carcinoembryonic antigen-related cell adhesion molecule cellular receptors. J Biol Chem 277: 19727-19734.

Navas S, Seo SH, Chua MM, Sarma JD, Lavi E, Hingley ST, Weiss SR (2001). Murine coronavirus spike protein determines the ability of the virus to replicate in the liver and cause hepatitis. J Virol 75: 2452-2457.

Peng D, Koetzner CA, McMahon T, Zhu Y, Masters PS (1995). Construction of murine coronavirus mutants containing interspecies chimeric nucleocapsid proteins. $J$ Virol 69: 5475-5484.

Perlman S, Jacobsen G, Olson AL, Afifi A (1990). Identification of the spinal cord as a major site of persistence during chronic infection with a murine coronavirus. Virology 175: 418-426.

Phillips JJ, Chua M, Seo SH, Weiss SR (2001). Multiple regions of the murine coronavirus spike glycoprotein influence neurovirulence. J NeuroVirol 7: 421-431.

Phillips JJ, Chua MM, Lavi E, Weiss SR (1999). Pathogenesis of chimeric MHV-4/MHV-A59 recombinant viruses: the murine coronavirus spike protein is a major determinant of neurovirulence. J Virol 73: 7752-7760.

Schmidt I, Skinner M, Siddell S (1987). Nucleotide sequence of the gene encoding the surface projection 
glycoprotein of coronavirus MHV-JHM. J Gen Virol 68 (Pt 1): 47-56.

Sorensen O, Coulter-Mackie MB, Puchalski S, Dales S (1984). In vivo and in vitro models of demyelinating disease. IX. Progression of JHM virus in the central nervous system of rat during overt and asymptomatic phases. Virology 137: 347-358.

Sturman LS, Ricard CS, Holmes KV (1985). Proteolytic cleavage of the E2 glycoprotein of murine coronavirus: activation of cell-fusing activity of virions by trypsin and separation of two different $90 \mathrm{~K}$ cleavage fragments. J Virol 56: 904-911.

Taguchi F, Matsuyama S (2002). Soluble receptor potentiates receptor-independent infection by murine coronavirus. J Virol 76: 950-958.

Wege H, Siddell S, ter Meulen V (1982). The biology and pathogenesis of coronaviruses. Adv Virol Immunol 99: 165-200.
Wege H, Stephenson JR, Koga M, Wege H, ter Meulen V (1981). Genetic variation of neurotropic and nonneurotropic murine coronaviruses. J Gen Virol 54: 6774.

Weiner LP (1973). Pathogenesis of demyelination induced by a mouse hepatitis virus (JHM virus). Arch Neurol 28: 298-303.

Yamada YK, Takimoto K, Yabe M, Taguchi F (1997). Acquired fusion activity of a murine coronavirus MHV-2 variant with mutations in the proteolytic cleavage site and the siganal sequence of the S protein. Virology 227: 215-219.

Yokomori K, Banner LR, Lai MC (1991). Heteroginity of gene expression of the hemagglutinin-esterase (HE) protein of murine coronaviruses. Virology 183: 647-657.

Yount B, Denison MR, Weiss SR, Baric RS (2002). Systematic assembly of a full-length infectious cDNA of mouse hepatitis virus strain A59. J Virol 76: 11065-11078. 